\title{
Accuracy of core needle biopsy diagnosis in assessing papillary breast lesions: histologic predictors of malignancy
}

\author{
Doina Ivan ${ }^{1}$, Veronica Selinko², Aysegul A Sahin ${ }^{3}$, Nour Sneige ${ }^{3}$ and Lavinia P Middleton ${ }^{3}$ \\ ${ }^{1}$ Department of Pathology, The University of Texas-Houston; ${ }^{2}$ Departments of Radiology and ${ }^{3}$ Pathology, \\ The University of Texas MD Anderson Cancer Center, Houston, TX, USA
}

\begin{abstract}
The purpose of this study was to determine the accuracy of core needle biopsy (CNB) diagnosis of papillary breast lesions and to identify histologic features that can predict malignancy. We retrospectively reviewed 2876 CNB performed at MD Anderson Cancer Center (01/95-08/02) and identified 50 papillary lesions: 30 papillomas, eight atypical papillomas and 12 papillary carcinomas. Histopathological parameters were evaluated and radiographic findings were reviewed. When available, the CNB was compared with the excisional biopsy (EB) material. Carcinoma was confirmed by EB in $11 / 12$ cases and invasion was correctly assessed in $67 \%$ of them. In EB, $6 / 8(75 \%)$ atypical papillomas revealed carcinoma in situ or atypia and the remaining two (25\%) were benign, six out of $30(20 \%)$ papillomas had been excised and none had shown atypia; the remaining patients had clinical and radiological follow-up with no evidence of disease progression. We conclude that CNB is effective for assessing papillary breast lesions and that EB is more accurate in determining invasion. Cellular monotony, lack of myoepithelial cells, and cytologic atypia are more accurate predictors of malignancy $(P<0.0001)$ than is the presence of mitoses $(P<0.053)$. A diagnosis of carcinoma or atypical papilloma by CNB should warrant an EB, whereas benign papillomas may be followed if imaging findings are concordant.
\end{abstract} Modern Pathology (2004) 17, 165-171, advance online publication, 19 November 2003; doi:10.1038/modpathol.3800014

Keywords: breast; papillary lesions; core needle biopsy; histology

Papillary lesions of the breast are commonly encountered in routine surgical pathology and consist of a heterogeneous group that includes papilloma, atypical papilloma, noninvasive papillary carcinoma, and invasive papillary carcinoma. Historically, open surgical biopsy was necessary to establish the histopathologic identity of these lesions. Recently, initial diagnosis by sonographically guided and stereotactic core needle biopsy (CNB) has replaced surgical excision in many institutions. ${ }^{1,2}$ Moreover, the subsequent decision about clinical management (follow-up vs surgical excision) is now based primarily on the CNB diagnosis. This emphasizes the importance of establishing reliable and accurate diagnostic criteria for these lesions. CNB is considered less invasive and diagnostically nearly as accurate as excisional biopsy (EB). However, in current pathology practice,

Correspondence: LP Middleton, Department of Pathology, The University of Texas MD Anderson Cancer Center, 1515 Holcombe Boulevard, Box 85, Houston, TX 77030, USA.

E-mail: lpmiddleton@mdanderson.org

Received 23 July 2003; accepted 8 October 2003; published online 19 November 2003 there are several difficulties regarding diagnosis and the subsequent management of papillary lesions encountered in the CNB specimens. Owing to the fragmented, limited material obtained by CNB, it may be extremely challenging to determine whether a lesion is benign or malignant and whether is noninvasive or invasive. ${ }^{3}$ Thus, CNB specimens are best interpreted with a knowledge of the lesion's imaging characteristics, both to ensure a pathologically accurate diagnosis and to assure the radiologist that the targeted lesion was adequately sampled. ${ }^{-3}$

The purpose of this study was to determine the accuracy of CNB diagnosis in evaluation of papillary breast lesions and to identify histopathologic and imaging features that can possibly be used as predictors of malignancy.

\section{Materials and methods}

We searched the pathology database at the University of Texas MD Anderson Cancer Center and retrospectively reviewed the results of 2876 consecutive imaging-guided CNB performed between January 1, 1995 and August 30, 2002 to identify 
cases with primary diagnosis of benign, atypical or malignant breast papillary lesions. In all, $50(1.7 \%)$ cases were identified including 30 papillomas (60\%), eight atypical papillomas (16\%) and 12 papillary carcinomas (24\%).

Two types of CNBs were evaluated in this study. Microcalcifications and architectural distortions had been sampled with stereotactic guidance using 11- or 14-gauge vacuum-assisted core biopsy (Mammotome; Ethicon EndoSurgery, Cincinnati, $\mathrm{OH}$, USA). Solid masses had been sampled with sonographic-guided 16-, 18- or 20-gauge cutting-needle biopsy (Biopty; Bard Urological, Covington, GA, USA). Of the 50 biopsies analyzed, 31 (62\%) had been performed under stereotactic guidance, and 19 $(38 \%)$ had been sampled under sonographic guidance.

The stereotactic biopsies had been performed with the patients in the prone position on a dedicated table (LoRad, Danbury, CT, USA). Between January 1995 and March 1997, 14-gauge cutting needles had been used while the patients were in an upright unit. The average number of vacuum-assisted biopsy specimens taken per patient was 11 (range, 5-22).

The sonographically guided biopsies had been performed with the patients in the supine or supine-oblique position by using a 7.5 or VFX 13$\mathrm{MHz}$ linear array transducer and high-resolution sonographic equipment (Sonoline, Siemens, Montainview, CA, USA) in a dedicated breast-sonography unit. The number of core samples taken had been left to the discretion of the breast radiologist performing the procedure and based on visual inspection of needle placement and the tissue sections obtained. The average number of sonographically guided specimens obtained per patient was 5 (range, 3-12).

Both stereotactic and sonographically guided biopsy specimens had been submitted in 1-5 blocks, and at least one hematoxylin and eosin (H\&E)stained glass slide of each block had been reviewed by two pathologists.

Histological parameters assessed included: presence or absence of myoepithelial cells, cytological and architectural atypia, cellular monotony, necrosis, and mitotic count. The association with calcifications and other breast lesions was also evaluated.

The lesions were categorized according to published histopathologic criteria. ${ }^{4-8}$ For the diagnosis of papilloma, we required proliferation of ductal epithelium with fibrovascular core formation, presence of myoepithelial cells and absence of pleomorphism or cellular monotony., ${ }^{4,8}$ Papillary proliferations with atypical monotonous epithelial cells and lacking myoepithelial cells were defined as papillary carcinoma. ${ }^{4,6,8}$ The diagnosis of atypical papilloma was reserved for those cases with focal cytological or architectural atypia, focal absence of myoepithelial cells and monotonous epithelial proliferation. ${ }^{8}$
In all cases of atypical papilloma, smooth muscle actin (SMA) stain to detect myoepithelial cells was performed: sections of each formalin-fixed, paraffinembedded tissue blocks were stained with monoclonal SMA (1:20) (Ventana Medical Systems, Tucson, AZ, USA) using steam heat-induced epitope retrieval and an avidin-biotin complex technique.

Whenever they were available, EB specimens were also examined and compared with the CNB specimen.

Mammographic and sonographic findings were reviewed by a dedicated breast radiologist (VS) to determine whether a mass, architectural distortion, or microcalcifications had been present. For cases in which a mass had been identified, the size, borders, and shape were evaluated. When microcalcifications were present, their type and distribution were noted.

We obtained clinical information from the medical records. The results of clinical or mammographic follow-up (mean, 16.7 months; range, 1-78 months) were also obtained from the clinical database.

The correlation between the assessed histopathologic parameters and their potential use as predictors of malignancy in the papillary breast lesions were analyzed using $\chi^{2}$ test and Fisher's exact tests, with $P<0.05$ considered to be statistically significant.

\section{Results}

\section{Clinical Characteristics}

Table 1 summarizes the characteristics of the 50 cases of papillary breast lesions that had been diagnosed by CNB. All of the patients were women between the ages of 31 and 89 years (mean, 59 years). The median age for the women with papillomas was 55 years, for atypical papillomas 58 years and papillary carcinomas 70 years. In total, $19(38 \%)$ of the women had a history of breast carcinoma in a first- or second-degree female relative documented in their medical records. Two (4\%) women had synchronous or metachronous breast carcinoma and eight (16\%) had other malignancies. Seven $(58 \%)$ patients with papillary carcinoma, four $(50 \%)$ with atypical papilloma, and 11 $(37 \%)$ with papilloma presented with palpable breast masses. Nipple discharge had been the chief complaint of two patients with papilloma, and a skin change (erythema or hyperemia) had been noted by one patient with papilloma and one with papillary carcinoma. Two patients from each category had described breast pain or tenderness.

\section{Radiographic Characteristics}

For all 50 cases a dedicated breast radiologist (VS) reviewed the mammograms and sonographic images and Table 1 summarizes their characteristics. 
Table 1 Clinical and imaging characteristics of the 50 patients with papillary breast lesions

\begin{tabular}{|c|c|c|c|}
\hline Characteristics & $\begin{array}{l}\text { Papilloma } \\
(\mathrm{n}=30)\end{array}$ & $\begin{array}{c}\text { Atypical } \\
\text { papilloma } \\
(\mathrm{n}=8)\end{array}$ & $\begin{array}{c}\text { Papillary } \\
\text { carcinoma } \\
(\mathrm{n}=12)\end{array}$ \\
\hline Median age at presentation & 55 & 58 & 70 \\
\hline \multicolumn{4}{|l|}{ Clinical presentation $^{\mathrm{a}}$} \\
\hline Pain & 2 & 2 & 2 \\
\hline Nipple discharge & 2 & 0 & 0 \\
\hline Skin change & 1 & 0 & 1 \\
\hline Palpable mass & 11 & 4 & 7 \\
\hline \multicolumn{4}{|l|}{ Mammographic findings } \\
\hline Mass present & 12 & 3 & 11 \\
\hline \multicolumn{4}{|l|}{ Borders } \\
\hline Circumscribed & 1 & 1 & 8 \\
\hline Indistinct & 11 & 2 & 3 \\
\hline \multicolumn{4}{|l|}{ Shape } \\
\hline Lobulated & 1 & 1 & 8 \\
\hline Round/oval & 0 & 0 & 2 \\
\hline Spiculated/irregular & 11 & 2 & 1 \\
\hline Associated microcalcifications & 22 & 6 & 4 \\
\hline \multicolumn{4}{|l|}{ Morphologic characteristics ${ }^{b}$} \\
\hline Pleomorphic & 13 & 2 & 2 \\
\hline Amorphous & 7 & 2 & 1 \\
\hline Coarse & 3 & 2 & 1 \\
\hline Punctate & 4 & 0 & 1 \\
\hline \multicolumn{4}{|l|}{ Distribution $^{\mathrm{b}}$} \\
\hline Clustered & 9 & 0 & 3 \\
\hline Segmental & 1 & 1 & 0 \\
\hline Linear/branching & 2 & 2 & 1 \\
\hline \multicolumn{4}{|l|}{ Sonographic findings } \\
\hline Mass present & 5 & 4 & 11 \\
\hline Solid & 3 & 2 & 8 \\
\hline Complex (cystic and solid) & 2 & 2 & 3 \\
\hline
\end{tabular}

Remainder of patients were asymptomatic.

${ }^{\mathrm{b}}$ More than one morphologic or distribution type of microcalcifications was encountered.

\section{Papilloma}

In $11(37 \%)$ of the 30 papillomas, mammography revealed a mass (range, $0.3-2.8 \mathrm{~cm}$; mean, $1.2 \mathrm{~cm}$ ) with indistinct and irregular borders. In one case $(3 \%)$, the mass was circumscribed and lobulated, and in another two $(6 \%)$ an architectural distortion was described. The ultrasound revealed a mass in five $(17 \%)$ cases and associated microcalcifications were identified in $22(73 \%)$ papillomas.

\section{Atypical papilloma}

Mammography revealed a mass in three (38\%) of the eight atypical papillomas; in one case the mass was well circumscribed and the other two had indistinct and irregular borders. The masses ranged from 1.0. to $1.2 \mathrm{~cm}$ in maximum dimension (mean, $1.1 \mathrm{~cm}$ ). Sonography identified a mass in four (50\%) cases of atypical papilloma. Associated microcalcifications were present in six $(75 \%)$ cases.

\section{Papillary carcinoma}

A mass was identified in 11 (92\%) of the 12 cases of papillary carcinoma, ranging from 0.7 to $10.0 \mathrm{~cm}$. (mean, $2.2 \mathrm{~cm})$. In eight $(73 \%)$ of these cases the
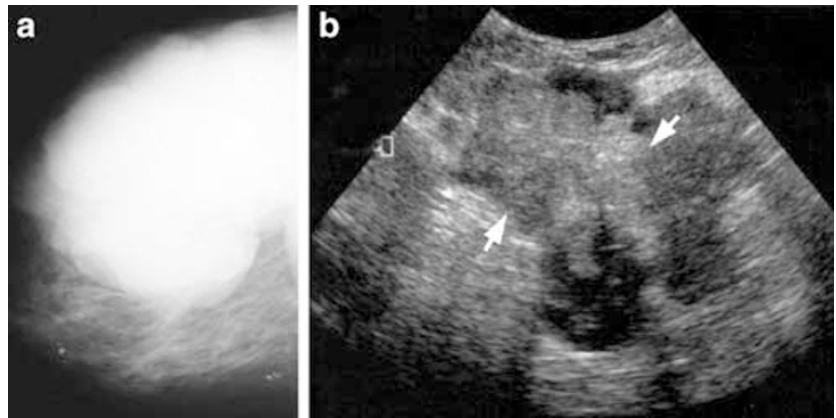

Figure 1 (a and b) Imaging findings in a patient with papillary carcinoma. The mammogram (a) shows a large, lobulated, relatively well-circumscribed mass in the upper outer quadrant. No calcifications are seen in association with the mass Sonography (b) demonstrates a complex mass (with both cystic and solid areas). Arrows indicate solid component.

mass was well circumscribed and lobulated (Figure 1); the remaining masses had indistinct borders. Sonography confirmed the mammographic findings for all 11 masses, eight of which were solid and hypoechoic and three were both cystic and solid. Associated microcalcifications were identified in four $(33 \%)$ of the 12 papillary carcinomas.

\section{Histopathologic Characteristics}

Table 2 summarizes the histopathologic findings of the specimens from the 50 patients with papillary breast lesions included in our study.

\section{Papilloma}

The histopathologic review of the CNB specimens from the 30 patients with benign papilloma showed the presence of ductal epithelial proliferation with papillary architecture, presence of myoepithelial cells, and the absence of cytological atypia and cellular monotony. No mitoses were identified in 24 $(80 \%)$ of the cases and one mitosis per 10 highpower fields (hpf) was counted in the other six $(20 \%)$ cases. Sclerosis and/or stromal hyalinization were encountered in $15(50 \%)$ cases (Figure 2). Associated microcalcifications were present in 21 $(70 \%)$ cases and were found within the proximity of the papilloma in another six $(20 \%)$ cases. Fibrocystic changes were also identified in 26 (87\%) specimens. EB had been subsequently performed in six $(20 \%)$ of the papillomas diagnosed on CNB; none of them showed atypia or malignancy. EB had been performed after the CNB diagnosis at an interval ranging from 1 month to 5 months (average 10 weeks) and was prompted in five cases by the presence of extensive fibrosis and hyalinization in the CNB specimen and in one case because the CNB diagnosis had not correlated with the mammographic findings. The remaining 24 cases had follow-up ranging from 1 month to 51 months (mean 14.7 months) with no clinical or radiological evidence of disease progression. 
Table 2 Histopathologic characteristics of the 50 papillary breast lesions

\begin{tabular}{|c|c|c|c|c|}
\hline $\begin{array}{l}\text { Histopathologic } \\
\text { characteristics }\end{array}$ & $\begin{array}{l}\text { Papilloma } \\
(\mathrm{n}=30)\end{array}$ & $\begin{array}{c}\text { Atypical } \\
\text { papilloma } \\
(\mathrm{n}=8)\end{array}$ & $\begin{array}{l}\text { Papillary } \\
\text { carcinoma } \\
(\mathrm{n}=12)\end{array}$ & P-value \\
\hline \multicolumn{5}{|l|}{ Architecture $^{\mathrm{a}}$} \\
\hline $\begin{array}{l}\text { Predominantly } \\
\text { papillary }\end{array}$ & 30 & 8 & 12 & \\
\hline Solid/cribriform & 1 & 2 & 8 & \\
\hline Architectural atypia & 0 & 2 & 2 & \\
\hline Cellular monotony & & & & $<0.0001$ \\
\hline Focal & 0 & 8 & 1 & \\
\hline Diffuse & 0 & 0 & 11 & \\
\hline Myoepithelial cells & & & & $<0.0001$ \\
\hline Present & 30 & 0 & 0 & \\
\hline Absent focally & 0 & 8 & 1 & \\
\hline Absent diffusely & 0 & 0 & 11 & \\
\hline Cytologic atypia & 0 & 7 & 12 & $<0.0001$ \\
\hline Necrosis & 0 & 0 & 2 & $<0.053$ \\
\hline \multicolumn{5}{|l|}{ Mitoses (per 10 hpf) } \\
\hline 0 & 24 & 4 & 5 & \\
\hline 1 & 6 & 3 & 5 & \\
\hline 2 & 0 & 0 & 1 & \\
\hline 3 & 0 & 1 & 1 & \\
\hline \multicolumn{5}{|l|}{ Nuclear grade ${ }^{b}$} \\
\hline 3 & & 0 & 2 & \\
\hline 2 & & 0 & 4 & \\
\hline 1 & & 7 & 6 & \\
\hline Not applicable & 30 & 1 & & \\
\hline Invasion & 0 & 0 & 5 & \\
\hline $\begin{array}{l}\text { Sclerosis/stromal } \\
\text { hyalinization }\end{array}$ & 15 & 4 & 2 & \\
\hline $\begin{array}{l}\text { Associated } \\
\text { microcalcifications }\end{array}$ & 27 & 6 & 4 & \\
\hline
\end{tabular}

a More than one architectural pattern was encountered in the same lesion.

${ }^{\mathrm{b}}$ We defined nuclear grade as follows: 3 , marked nuclear pleomorphism, irregular nuclear contour, coarse chromatin and multiple nucleoli; 2, mild to moderate nuclear pleomorphism, one or two nucleoli, high N/C ratio; 1, monomorphic nuclei with inconspicuous nucleoli.

\section{Atypical papilloma}

In all eight cases of atypical papilloma a predominantly papillary architecture was identified, and in two of the cases an associated cribriform or solid pattern was also present. Focal cytological or architectural atypia, appearing as expansion of papillae, prominent nucleoli or nuclear hyperchromasia, were a common finding for all of the atypical papillomas (Figure 3). Focal cellular monotony was also identified. Myoepithelial cells, highlighted by SMA immunostain, were at least focally present in all cases (Figure 4). The mitotic count was 3 mitoses/10 hpf in one case, 1 mitosis/10 hpf in three cases and zero in the remaining four cases. Necrosis was not a feature of the atypical papillomas in our series. Sclerosis and/or stromal hyalinization were identified in four $(50 \%)$ of the specimens. Microcalcifications were identified in six $(75 \%)$ CNB showing atypical papilloma and associated fibrocystic changes were present in seven $(88 \%)$ cases. EB had been performed for all eight patients with atypical papilloma at an interval ranging from 3 weeks to 9 months (average 10 weeks) after CNB

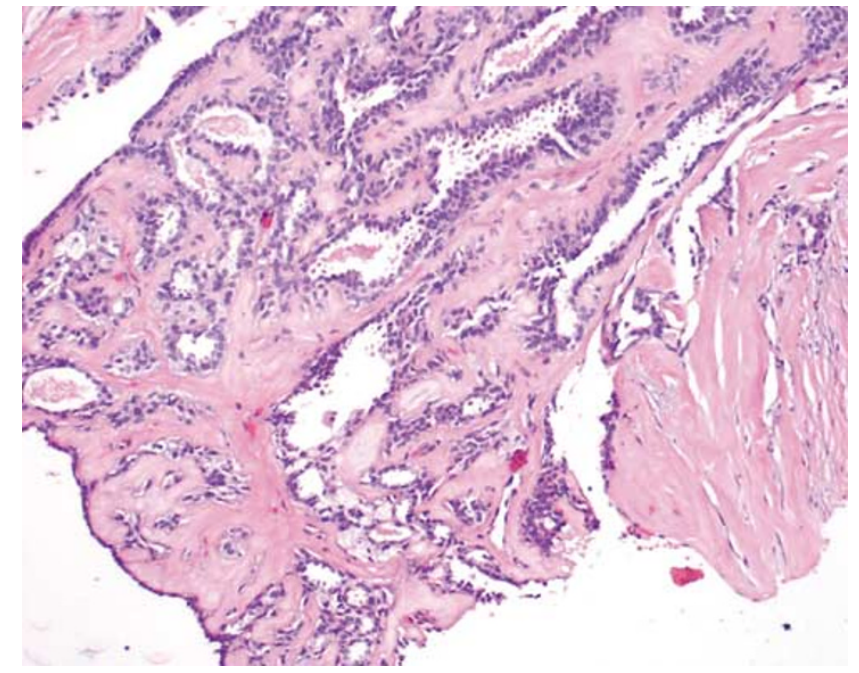

Figure 2 Papilloma with sclerosis or stromal entrapment of epithelial cells.

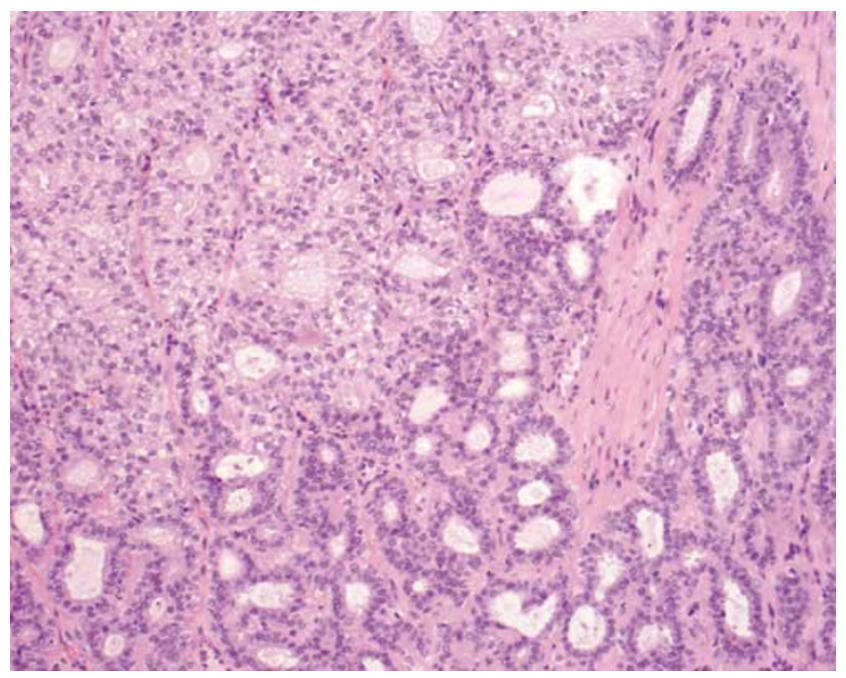

Figure 3 Atypical papilloma diagnosed on CNB, revealing a monotonous cellular proliferation with expansion of the papillae (left side).

diagnosis. EB revealed carcinoma in situ in five $(63 \%)$ cases, atypical ductal hyperplasia in one $(12 \%)$ case, and benign papilloma or no residual disease in the remaining two $(25 \%)$ cases.

\section{Papillary carcinoma}

Examination of the CNB from the 12 patients with papillary carcinoma revealed common features (Figure 5): cellular monotony, atypical cytology, and lack of myoepithelial cells $(P<0.0001)$. The papillary architecture was present in all cases; in eight $(67 \%)$ cases, an associated solid or cribriform architecture was also identified. The mitotic count per $10 \mathrm{hpf}$ was 3 in one case, 2 in another one case and 1 and zero for each of the remaining five cases. Necrosis was present in only two $(17 \%)$ cases $(P<0.053)$. Associated microcalcifications were 


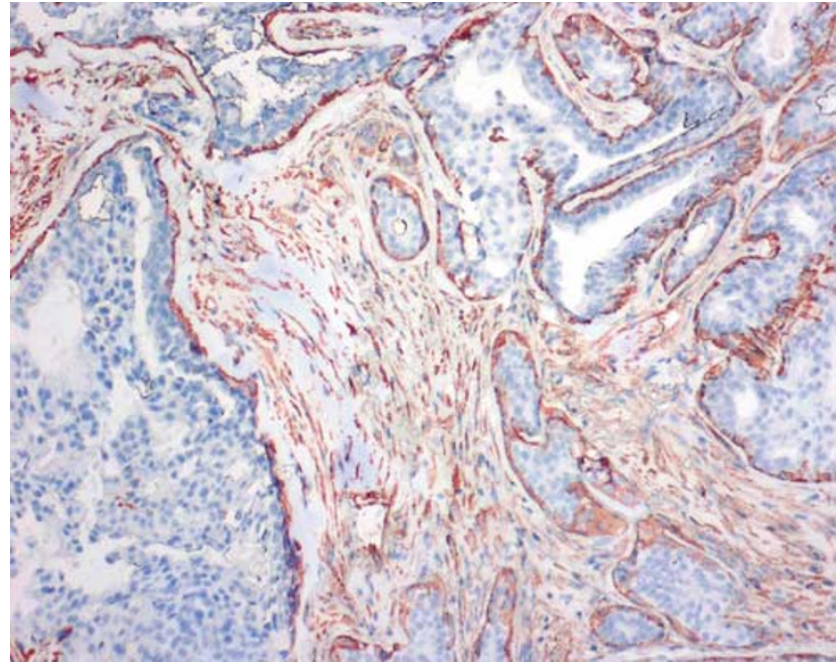

Figure 4 Atypical papilloma on CNB. The smooth muscle actin (SMA) highlights the myoepithelial cells in the benign area of this section (right) whereas it is negative in areas with monotonous cell proliferation and cytological atypia (left).

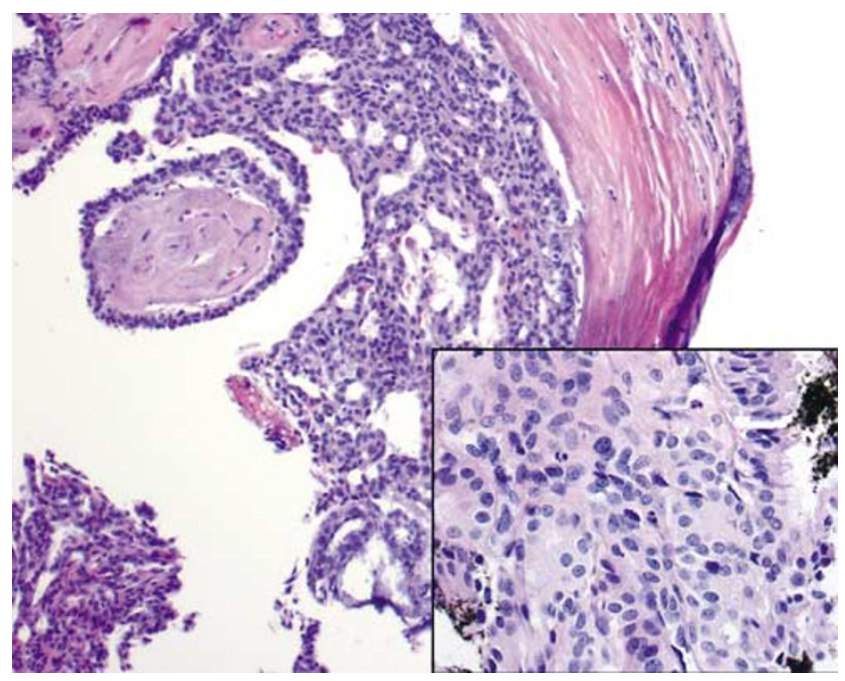

Figure 5 Papillary carcinoma on CNB demonstrating a monotonous cellular proliferation with papillary architecture and nuclear hyperchromasia. The inset is a higher power of the carcinoma and reveals cellular pleomorphism and numerous mitoses.

present in four (33\%) cases. In three (25\%) cases, associated ductal carcinoma in situ (DCIS) was identified. Subsequent EB had been performed in 11 of the 12 cases at an interval ranging from 2 weeks to 3 months (mean, 5 weeks) after the CNB, confirming the diagnosis. One patient had deferred excision because of a history of colon carcinoma and associated lung and liver metastases. Invasion had been correctly assessed by CNB in eight (67\%) cases. EB specimens also revealed associated DCIS in nine $(75 \%)$ cases and benign lesions (ie fibrocystic changes, fibroadenoma) in four (33\%) patients.

In all cases of papilloma, atypical papilloma, and papillary carcinoma, correlation between the histo- pathologic findings with radiographic abnormalities confirmed correct identification of the targeted lesions.

\section{Discussion}

The use of stereotactic or ultrasound guided percutaneous CNB for the initial evaluation of clinically occult breast lesions, including lesions with a predominant papillary growth pattern, has evolved to a practical alternative to EB in many institutions. ${ }^{1-3}$ Considering that the subsequent decision about clinical management (follow-up vs surgical excision) is now based primarily on the CNB diagnosis, there is an increased interest in establishing reliable and accurate diagnostic criteria for these lesions.

Recently, Jacobs et $a l^{3}$ underlined that no published studies have identified histopathologic features of papillary breast lesions on CNB that could predict the presence of a more advanced lesion on excision. ${ }^{3}$ The present study is focused primarily on assessing the accuracy of CNB diagnosis in evaluation of papillary breast lesions and identifying possible histologic and radiological features that can aid in predicting malignancy.

Common histopathologic characteristics have been described for both benign and malignant papillary breast lesions. ${ }^{4-8}$ As emphasized by Azzopardi, ${ }^{4}$ the hallmark is a papillary, arborescent growth pattern supported by fibrovascular stalk. The single most valuable histologic feature distinguishing malignant from benign papillary breast lesions was found to be the presence of atypical epithelial proliferation of a single cell type. ${ }^{8}$ Papillary carcinoma lacks the continuous, basally oriented myoepithelial cell layer, which is considered the hallmark of benign papillomas. The histological identification of such myoepithelial cells is based mainly on the ovoid shape, clear cytoplasm and darkly stained nucleus. However, because of their many different morphologic characteristics, myoepithelial cells are not easily identified on H\&Estained sections. Immunohistochemical staining for SMA is a valuable addition to morphological findings, allowing a correct identification of the actin-rich myoepithelial cells. Markers such as epithelial membrane antigen (EMA), gross cystic disease fluid protein (GCDFP 15), calponin, and CD $44 \mathrm{~s}$ have been used in various studies, but none independently permitted the distinction between benign and malignant papillary lesions. ${ }^{9-11}$

\section{Papilloma}

The patients with papillomas were the youngest in our study (median age, 55 years) and the majority $(73 \%)$ presented with calcifications on mammography. The benign papillary lesions of the breast sampled by CNB were more likely associated with 
sclerosis, stromal hyalinization or fibrocystic changes than the atypical papillomas or papillary carcinomas. When the patients diagnosed with papilloma on CNB underwent excision, there was an excellent correlation between the CNB and EB findings. The main reasons for benign papillomas to be excised were: sclerosis or stromal hyalinization with epithelial entrapment (five cases), and because the CNB diagnosis did not correlate with the mammographic findings (one case). The remainder of the patients diagnosed with papillomas on CNB had been followed clinically and radiologically; no evidence of disease progression was found in this group.

\section{Atypical Papilloma}

The atypical papillomas are generally less precisely defined. In our study, the median age of the patients was intermediate between papillary carcinoma and benign papilloma and the radiological appearance of these lesions was mixed. The diagnosis of atypical papilloma was made on CNB specimens that had focal cytological or architectural atypia. In EB specimens, atypical papilloma is defined by the presence of atypical features in less than $1 / 3$ of the lesion $^{8}$ or in less than $4 \mathrm{~mm}$ of the specimen. ${ }^{12}$

In evaluating CNB specimens, we found that the atypia could be present only focally; involvement of a certain percentage of the lesion was not required in order to warrant a recommendation for EB. SMA immunostaining aided in the interpretation of atypical papillomas by highlighting the focal expansion of the papillae and the absence of myoepithelial cells. Our study found very good correlation between CNB and EB in diagnosing atypical papilloma. Authors who have investigated atypia in papillomas on incisional biopsy and correlated it with EB have found similar results. ${ }^{12}$ In the current study, EB revealed carcinoma arising in papilloma or atypical papilloma in six $(75 \%)$ of the cases, sclerotic papilloma in one $(12 \%)$ case, and only a biopsy cavity was identified in the remaining one case.

\section{Papillary Carcinoma}

In our study, the median age for women with papillary carcinoma was 15 years older than for women with benign papilloma. Interestingly, the mammography revealed a mass in $92 \%$ of papillary carcinoma cases and they tended to be wellcircumscribed, lobulated and larger than those found in patients diagnosed with benign or atypical papilloma. Associated microcalcifications were less commonly associated than in papilloma cases. We had excellent correlation in EB for the patients diagnosed with papillary carcinoma on CNB; the diagnosis was confirmed on EB in all examined cases. The presence of cellular monotony, cytological atypia and lack of myoepithelial cells in CNB specimens were accurate predictors of carcinoma $(P<0.0001)$. In addition to the predominantly papillary pattern, the associated solid or cribriform architecture was more likely present in CNB specimens from malignant lesions than those from benign lesions.

We can conclude that there are some histopathological features helpful in differentiating papillary lesions on CNB. Cellular monotony, lack of myoepithelial cells, and cytological atypia in CNB specimens are accurate predictors of carcinoma and atypical papilloma $(P<0.0001)$. In our experience, although all cases of papillary carcinoma and atypical papilloma yielded mitoses, six of the 30 papillomas also revealed 1 mitosis/10 hpf. Therefore, using mitoses as a predictive factor of malignancy is less accurate $(P<0.053)$ than cellular monotony or cytological atypia. Necrosis, when present, is a helpful feature for diagnosing malignancy. However, it was identified in only two of our 12 cases of papillary carcinoma and thus could not be used as a statistically significant discriminating criterion. SMA immunohistochemical staining can be also helpful in evaluating papillary lesions on CNB.

There are several studies suggesting that the presence of multiple intraductal papillomas might be an indicator of increased likelihood of evolution into malignancy. ${ }^{13}$ However, in our study, we found no definite evidence of histopathologic or prognostic differences between central and peripheral or between single and multiple papillary lesions.

As in all evaluations of the breast specimens obtained by CNB, radiologic and histopathologic correlation is essential. ${ }^{14-16}$ Discordant imaging and histopathologic findings should prompt a repeat biopsy and a joint review of lesions is recommended.

When a papillary lesion is suspected radiologically, it may be prudent to sample both the center and periphery of the lesion, as an invasive papillary lesion is most readily identified by sampling the periphery.

In summary, our study shows an excellent correlation between the CNB and EB in the diagnosis of benign and malignant papillary breast lesions. Therefore, we believe CNB is an effective and accurate tool in the assessment of these lesions. Based on our experience, the diagnosis of papilloma on CNB is reliable when correlated with imaging findings. Although the follow-up time in our series is relatively short (mean, 14.7 months), we recommend that these lesions, with good histopathologicradiologic correlation, should have a close mammographic and clinical follow-up instead of EB. All papillary carcinoma cases diagnosed on CNB should warrant subsequent EB. In our study, the diagnosis of invasion was most accurately determined after reviewing the EB material; invasion was correctly assessed by CNB in only six (67\%) of 11 cases of papillary carcinoma. The patients diagnosed with 
atypical papilloma on CNB should also have EB, as the majority of these lesions (six out of eight in our study) will likely reveal atypia or carcinoma in situ in the excised breast tissue.

\section{References}

1 Liberman L, Bracero N, Vuolo MA, et al. Percutaneous large-core biopsy of papillary breast lesions. Am J Roentgenol 1999;172:331-337.

2 Rosen EL, Bentley RC, Baker JA, et al. Imaging-guided core needle biopsy of papillary lesions of the breast. Am J Roentgenol 2002;179:1185-1192.

3 Jacobs TW, Connolly JL, Schnitt SJ. Nonmalignant lesions in the breast core needle biopsy; to excise or not to excise? Am J Surg Pathol 2002;26:1095-1110.

4 Azzopardi JG. Papilloma and papillary carcinoma. In: Azzopardi JG (ed), Problems in Breast Pathology. WB Saunders: Philadelphia, PA, 1979, pp 150-166.

5 Kraus FT, Neubecker RD. The differential diagnosis of papillary tumors of the breast. Cancer 1962;15: 444-455.

6 Rosen PP. Papillary carcinoma. In: Rosen PP (ed), Rosen's Breast Pathology. Lippincott-Williams \& Wilkins: Philadelphia, PA, 2001, pp 381-404.

7 Rosen PP. Papilloma and related benign tumors. In: Rosen PP (ed), Rosen's Breast Pathology. LippincottWilliams \& Wilkins: Philadelphia, PA, 2001;77-120.
8 Tavassoli FA. Papillary lesions. In: Tavassoli FA (ed), Pathology of the breast. Appleton \& Lange: Norwalk, CT, 1999;325-372.

9 Mosunjac MB, Lewis MM, Lawson D, et al. Use of a novel marker, calponin, for myoepithelial cells in fineneedle aspirates of papillary breast lesions. Diagn Cytopathol 2000;23:151-155.

10 Papotti M, Eusebi V, Gugliotta P, et al. Immunohistochemical analysis of benign and malignant papillary lesions of the breast. Am J Surg Pathol 1983;7:451-461.

11 Saddik M, Lai R. CD44s as a surrogate marker for distinguishing intraductal papilloma from papillary carcinoma of the breast. J Clin Pathol 1999;52:862-864.

12 Page DL, Salhany KE, Jensen RA, et al. Subsequent breast carcinoma risk after biopsy with atypia in a breast papilloma. Cancer 1996;78:258-266.

13 Ali-Fehmi R, Carolin K, Wallis T, et al. Clinicopathologic analysis of breast lesions associated with multiple papillomas. Hum Pathol 2003;34:234-239.

14 Cardenosa G, Eklund GW. Benign papillary neoplasms of the breasts: mammographic findings. Radiology 1991;181:751-755.

15 Rosen PP, Liberman L, Dershaw DD. Benign papillary tumors. In Rosen PP, Liberman L, Dershaw DD (eds), Breast Pathology-Diagnosis by Needle Core Biopsy. Lippincott-Williams \& Wilkins: Philadelphia, PA, 1999, pp 21-42.

16 Soo MS, Williford ME, Walsh R, et al. Papillary carcinoma of the breast: imaging findings. Am J Roentgenol 1995;164:321-326. 